\title{
Dynamic choices are most accurate in small groups
}

\author{
Julián Vicente-Page $^{1,2}$ • Alfonso Pérez-Escudero ${ }^{2,3}$ • Gonzalo G. de Polavieja ${ }^{1,2}$ (i)
}

Received: 8 June 2017 / Accepted: 6 October 2017 / Published online: 30 October 2017

(C) The Author(s) 2017. This article is an open access publication

\begin{abstract}
According to the classic results of Galton and Condorcet, as well as in modern decision-making models, accuracy in groups increases with group size. However, these studies do not consider the naturally occurring situation in which individuals dynamically re-evaluate their decision with a possible change of opinion. The dynamics of re-evaluation in groups are very different to individual re-evaluation because individuals influence the group and the group influences the individual. We find that individual accuracy in a group is higher when individuals re-evaluate because all members have more access to social information, while in single decisions, those deciding first have less. This improvement is smaller in large groups as in this case errors can cascade across the members of the group before re-evaluation can correct them. The net result is a maximal accuracy at a small group size. We also analyzed the case in which individuals are influenced only by a small number of the other individuals. In this case, cascading errors affect the interacting subgroups but are very unlikely to reach the whole group. This results in a local optimum at a small group size but also an optimum at a very large size. We thus
\end{abstract}

Gonzalo G. de Polavieja

gonzalo.polavieja@neuro.fchampalimaud.org

1 Champalimaud Neuroscience Programme, Champalimaud Center for the Unknown, Lisbon, Portugal

2 Instituto Cajal, Consejo Superior de Investigaciones Científicas, Madrid, Spain

3 Physics of Living Systems, Physics Department, MIT, Cambridge, MA, USA suggest that re-evaluation dynamics can make small and very large groups optimal. Also, features that may be seen as limitations, like an influence from only a small number of individuals, may turn to be beneficial when considering local animal interactions, here filtering out cascading of errors in the group when reconsideration dynamics takes place.

Keywords Collective behavior - Collective decisions . Dynamic decisions

\section{Introduction}

Animal group sizes in nature range from small groups of lions to very large bird flocks or fish schools (Krause and Ruxton 2002; Sumpter 2010; Packer et al. 1990; Cockburn 1998; Bonabeau et al. 1999; Niwa 1998). Each individual in any of these groups is exposed to social information that allows them to react better to the environment. However, an understanding of how decision accuracy depends on group size is still incomplete despite a long history of work in decision-making in groups. The classical results of the Condorcet jury theorem (Condorcet JANdC 1785) and group estimation experiments by Galton (1907), known popularly as wisdom of the crowds (Surowiecki 2004), point to very large groups as the most accurate. The intuition behind these classic results is that individuals are noisy estimators of features in the environment and that the larger the group, the easier to cancel out the noise in their estimations. Modern decision-making models in animal groups are based on similar ideas (Ward et al. 2008; Sumpter et al. 2008; PérezEscudero and de Polavieja 2011; Arganda et al. 2012) and also point to large groups as the most accurate. 
An important difference between the classic studies of Galton and Condorcet and modern group decision-making models is that, in the new models, agents do not decide independently but instead interact with each other. These interactions have been modeled, for example, as sequential decisions of rational agents using their private information and the estimations of previous agents. These models have shown that initial errors by very few members can easily propagate to the group in what is called an information cascade, although they are fragile with respect to new private information (Bikhchandani et al. 1992; Banerjee 1992). Interactions are also at the core of voting models. Here, agents update the value of an opinion variable depending on the opinion of others. Using techniques from network theory and statistical physics, it is studied how the population reaches a consensus decision (Castellano et al. 2009; Boccaletti et al. 2006). Other voting models allow the population to converge to several subgroups with different opinions (Shao et al. 2009; Havlin and Bunde 1991; Stauffer and Aharony 1994).

Recently, small group sizes have been obtained as the most accurate in a consensus model (Kao et al. 2014). In this case, individuals in a group are confronted with two options and also two cues, one cue of high and another of low correlation. Each individual chooses a cue with a given probability. Each of those agents chooses the low correlation option then chooses with a given probability the correct option. In contrast, all those agents choose the high correlation cue then choose the same option but also with a given probability. The choice made by the whole group is a consensus decision by majority voting. This model teaches us the important lesson that decisions can impose correlations among individuals that make large groups inaccurate.

Here, we reconsider the models obtained to explain animal decisions in experiments using groups (Sumpter et al. 2008; Ward et al. 2008; Pérez-Escudero and de Polavieja 2011; Arganda et al. 2012). We will use the same equations tested in these experiments, but we point to an implicit assumption in how they have been used. Their application has been restricted to the case of animals deciding in a single step. In nature, however, it should be very common to have some dynamics of reconsideration of the decisions, given that there is typically some time between the moment animals first perceive a scenario and the moment when they actually need to finally commit to one of the options. Our scenario differs from information cascades, voting models, and Kao et al. (2014) in that it considers empirical decisions rules obtained in animal groups. Respect to information cascades (Bikhchandani et al. 1992; Banerjee 1992), it shares the importance of cascades of errors, but we consider empirically tested decision rules and add reconsideration dynamics. Respect to voting models (Castellano et al. 2009; Boccaletti et al. 2006; Shao et al. 2009; Havlin and Bunde
1991; Stauffer and Aharony 1994), we share reconsideration dynamics, which in voting rules appears natural in the updating dynamics until convergence to global consensus states. With Kao et al. (2014), we share the importance of correlations among individuals in the group to give small groups as those of optimal size, but we do not consider cues or majority voting.

We show that the dynamics in which individuals in groups can reconsider their choices leads naturally to the result that groups of small size choose best. Re-evaluation allows a better access to social information to all members in the group, resulting in a better decision accuracy but less so for large groups in which re-evaluation does not stop chains of errors. The highest accuracy then corresponds with groups of intermediate size, and this optimal size decreases as the influence of the social information increases. These results are obtained for many different models as long as they share basic properties common in animal collectives: (a) an individual is more likely to do a behavior if others are already doing it, (b) not all individuals decide at the same time, and (c) decisions are dynamical so there can in principle be changes in opinion.

We also studied the case in which each individual is only influenced by a small number of individuals in the group. The results in this case are similar at small or intermediate group sizes as the number of influencing individuals is still not too dissimilar from the total number in the group. For large groups, however, the information cascades still happen at the level of the subgroups but very unlikely affect the whole group. For this case, we then obtain small group sizes as locally optimal but good accuracy at large sizes as well.

\section{Results}

\section{Decision-making with re-evaluation}

We considered individuals in a group re-evaluating their decisions (Fig. 1a). We first used a toy scenario for simplicity of analysis and then tested that results hold for more realistic scenarios. We considered a group of agents deciding between left (say, correct option) and right (incorrect) in a fixed sequence and in rounds of re-evaluation. This clean separation into rounds and into strict sequences is the artificial part of the scenario that simplifies analysis, but we will see later that it is not necessary for the result.

In the first round, agent 1 decides using only its private information about the options. Then, agent 2 decides using its private information and the fact that animal 1 has chosen left. The same for animals 3 to 8 , who also decide based on private information and the decisions of other animals (Fig. 1a, first round). In this particular example, all animals except 2 and 4 choose left. This finishes the first round, a 

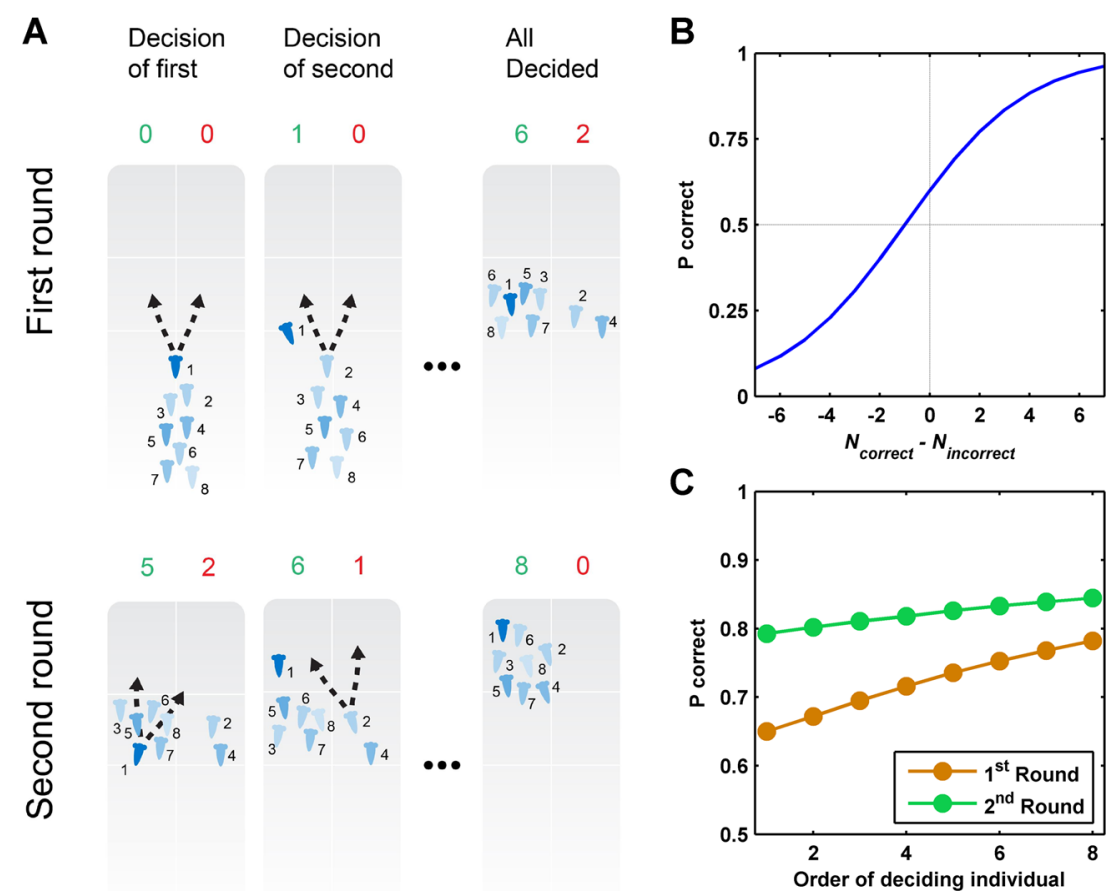

Fig. 1 Decision-making in collectives with re-evaluation of choice. a Simple example of re-evaluation of choices. Individuals are represented by different colors and their deciding orders are also highlighted. In the first round, agents decide in a sequence according to their private information of which side is the best (left or right) and on their social information. At each step, a number of individuals choosing each option are shown above (green: left, correct option; red: right, incorrect option). In the second round, there is a new sequence of decisions in the same order in which each agent re-evaluates its choice. $\mathbf{b}$ Probability of choosing the correct side, as a function of the difference

scenario commonly modeled in decision-making in groups (Ward et al. 2008; Sumpter et al. 2008; Pérez-Escudero and de Polavieja 2011; Arganda et al. 2012). Note that the agents that decided first in this round had less access to social information.

In a second round, all agents re-evaluate their choices again in the same sequence $1,2, \ldots, 8$. In the example illustrated here, animals 2 and 4 change their choices by the weight of the social information (Fig. 1a, second round). In this second round, all animals have access to a richer social information than in the first round, especially those that chose first.

\section{Re-evaluation implies the highest accuracy at intermediate group size}

In the following, we use our toy scenario to show that groups of intermediate size are more accurate. Before using a mathematical model to show this result, it is instructive to advance the intuition.

One part of the result is that re-evaluation allows access to social information for those that chose first without between the number of individuals that previously chose each option (see Eq. 1). With better private information (parameter $a$ in Eq. 1, here fixed to $a=1.5$ ), the curve moves to the left so less social information is needed to make a correct choice. With higher influence of social information (parameter $s$ in Eq. 1, here fixed to $s=1.5$ ), the higher the slope and the lesser the other individuals one needs to take into account to have a high probability of making the correct choice. $\mathbf{c}$ Probability per individual of choosing the correct option at the first and at the second round. The results are obtained by applying the formula at $\mathbf{b}$ to the general case of a group of eight individuals

access to this information. The second part is that large groups cannot improve in re-evaluation because of error propagation. To see this, consider that the first two agents in the first round choose the wrong option. These wrong choices make the remaining agents in the group more likely to make mistakes as well. If the group is small, when agent 1 decides in the second round, it does not find a very large number of individuals in error and it still has a reasonable probability of correcting. In contrast, when the group is large, by the time agent 1 makes a decision in the second round, the number of individuals making a wrong decision is large, and therefore, agent 1 is unlikely to correct its choice using social information.

The intuition is then that while an extra round helps to obtain more social information, errors are more difficult to correct using social information in the larger group.

For a quantitative treatment, we started with a simple model that matches the toy situation illustrated in Fig. 1a. This model is an extension (to the case of several decision rounds) of a model we successfully applied to collective decision-making in sticklebacks (Pérez-Escudero and de Polavieja 2011) and also adapted to humans (Madirolas and 
de Polavieja 2015). In the context of a two choice setup, each animal has a probability of choosing the correct option in the form

$$
P_{c}=\frac{1}{1+\frac{1}{a}\left(\frac{1}{s}\right)^{N_{c}-N_{i}}},
$$

illustrated in Fig. $1 \mathrm{~b}$ for parameter values $a=s=1.5$. From this plot, it is clear that the probability of making the correct choice increases with how many more animals have already chosen correctly than incorrectly, $N_{c}-N_{i}$. It also increases with the quality of private information $a$ (the curve shifts to the left) and the influence of social information provided by each other animal $s$ (the slope increases).

Application of the probability in Eq. 1 to a group deciding in a sequence is as follows. The first individual to decide in the first round would choose according to Eq. 1 using private information $a$ and with no other animal having made any choice, $N_{c}-N_{i}=0-0=0$, giving $P_{c}=a /(a+1)$. If using this probability, animal 1 chooses, say, to move to the correct option then the second animal would choose according to Eq. 1 but now with $N_{c}-N_{i}=1-0=1$, that is $P_{c}=(1+(1 / a)(1 / s))^{-1}$. If animal 2 uses this probability and chooses, say, the wrong option then the third one again uses (1) with $N_{c}-N_{i}=1-1=0$ and so on until individual 8. This can leave the group in, for example, a configuration $\left(N_{c}, N_{i}\right)=(6,2)$ (Fig. 1a, first round).

We can compute for our example the mean probability of being correct in the first round as a function of the position of the animal in the decision sequence of the first round (Fig. 1c, orange; see Methods for details). The later the individual in the chain of choices the more access to social information, which makes them more likely to choose the correct option.

In a second round, the individuals re-evaluate the private and social information to make new decisions. For our first analysis, we assume the order animals deciding is the same order than in the first round, animals 1 to 8 . The only difference between this second round and the first one is that the starting configuration in the second round is the last configuration in the first round, in our example $\left(N_{c}, N_{i}\right)=(6,2)$. We also computed for this second round the probability that an animal makes a correct choice as a function of the order of decision (Fig. 1c, green; see Methods for details). All individuals improve in the second round, but more the ones deciding first.

We then asked what is the accuracy, computed as probability of being correct averaged across individuals in the group, depending on group size and number of decision rounds (Fig. 2a). This figure was obtained for the particular values of parameters $a=s=1.5$ in Eq. 1. For a decision with no re-evaluation, the larger the group, the better the choice (Fig. 2a, round = 1). In contrast, re-evaluation causes intermediate-sized groups to be most accurate (Fig. 2a, round $\geq 2$ ). For a high number of re-evaluations, the optimal size quickly converges to a size of 8 .

So far, we have used our model (1) with a particular set of parameters, private information $a=1.5$, and social information $s=1.5$. We then asked about the impact of the private and social information. Increasing the private information $a$ increases accuracy at optimal group size (Fig. 2b) and increases slightly the size of the optimal group (Fig. 2c, center). Increasing social influence $s$ implies higher accuracy at optimal size in the interval $s=1-1.5$ and $a>1.3$ and a small decrease elsewhere (Fig. 2b). Increasing the social influence $s$ very rapidly decreases the size of the optimal group (Fig. 2c, center). Except for the limit of very low social information ( $s$ close to 1 ), the best groups are those at intermediate sizes of around $2-14$ individuals. For example, for sticklebacks, we found elsewhere that $s=2-3$ (Pérez-Escudero and de Polavieja 2011) and optimal group sizes with re-evaluation would then correspond to groups of $4-6$ members.

It is important to also note that accuracy is asymmetric around the optimal group size (Fig. 2a). Groups smaller than optimal lose accuracy faster than groups larger than optimal. This is the same for any values of the model parameters (Fig. 2c).

\section{Mechanism underlying the highest accuracy at intermediate group size}

Our next question was how the optimal group size comes about. We have already seen that re-evaluation improves accuracy for all members of the group (Fig. 1c) and for all group sizes (Fig. 2a). However, in the following, we show that the benefit of re-evaluation is smaller for large groups. To see this in some detail, we studied what is the effect of the first two animals making the wrong choice in the first round (Fig. 3a). Specifically, we computed the probability that the first individual chooses the correct option at each subsequent round after the first two individuals chose incorrectly in the first round. In small groups, say a group of 4 , the first individual in the second round still has a high probability of choosing correctly because the number of incorrect choices in the first round is at most as large as the group size of 4 (Fig. 3a, blue, group size $=4$ ). In larger groups, say 12 individuals in this example, the first animal choosing in the second round has a much lower probability of choosing correctly (Fig. 3a, blue, group size $=12$ ). This is because it often finds quite a few animals having made the wrong choice and the social information strongly favors less the correct option.

We obtained more detail studying the proportion of the group that has made a correct choice (Fig. 3b). In the first 
Fig. 2 Re-evaluation implies the highest accuracy at intermediate group size. a Probability of choosing the correct option for several group sizes and for different number of rounds. b The highest possible accuracy achieved by groups of animals depending on influence of private and social information. $\mathbf{c}$ Group size with the highest accuracy depends on influence of private and social information, $a$ and $s$, respectively. The achieved accuracy is shown in $\mathbf{b}$. Four points in these parameters are chosen to show the maximum, $a=1.5$ and $s=3$ (top left), $a=3.6$ and $s=2.5$ (top right), $a=1.8$ and $s=1.4$ (bottom left), $a=3.7$ and $s=2.8$ (bottom right)
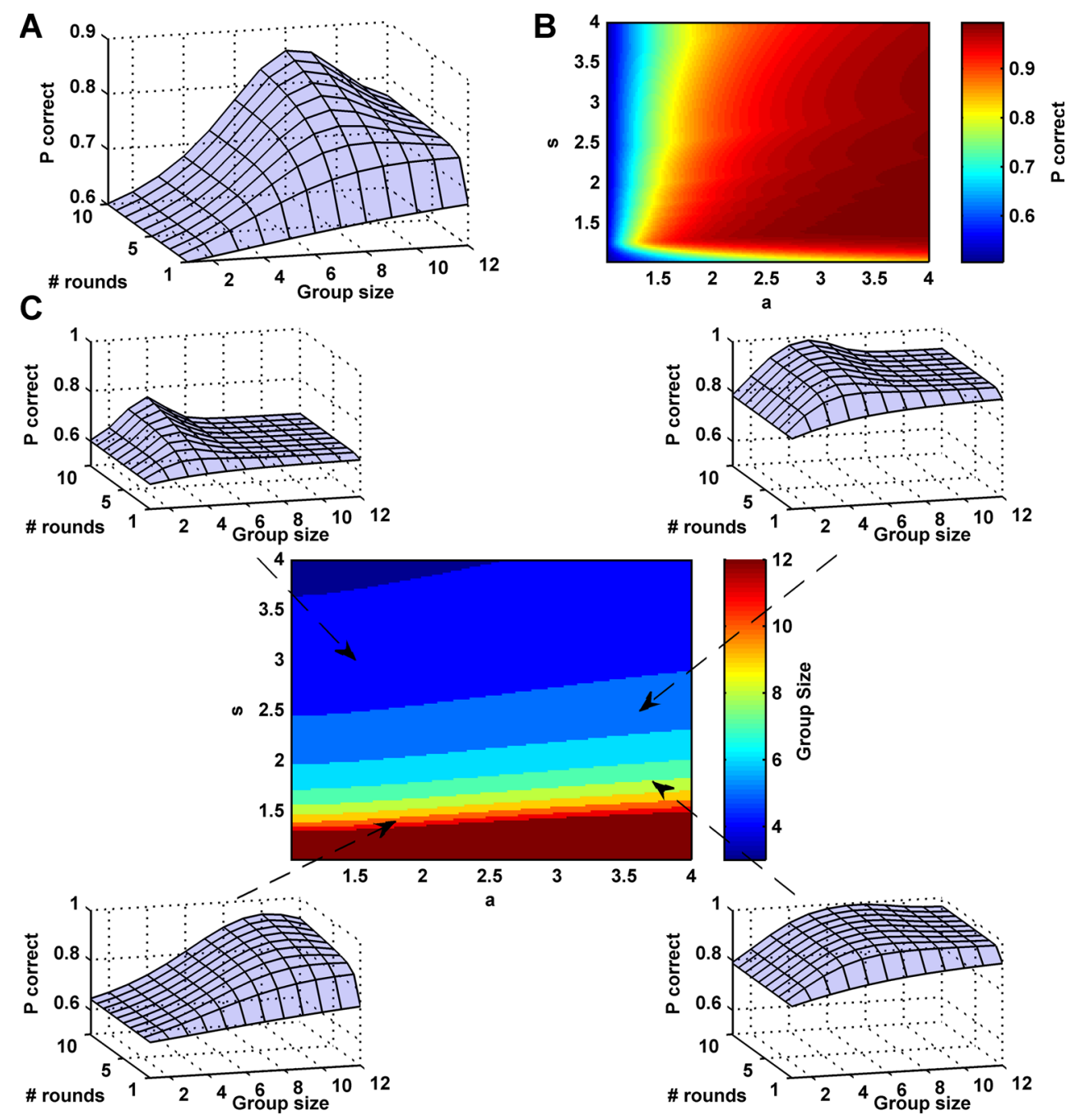

round, a group of 4 (left) or 8 (middle) has as it most probable configuration one with all animals choosing the correct option, and as its least probable configuration the one with all choosing incorrectly (Fig. $3 b$, round $=1$ ). For a group of 12 , the highest probability is again for the configuration with all the group choosing correctly, but now the configuration with all members choosing incorrectly is not the least probable. For the group of 4 , the re-evaluation dynamics shifts the probability slightly to configurations with more members of the group correct but still with a high probability of configurations in which a large proportion are incorrect. For a group of 8, the re-evaluation dynamics strongly shifts the probability to configurations with most individuals in the group choosing correctly. For a group of 12 , re-evaluation makes the probability bimodal, with the highest probability that all individuals are correct but also with a high probability of all incorrect. Large groups are very cohesive at the price of a high probability of all choosing the incorrect option. On average, an individual is more likely to choose correctly in a group of 8 in this example.

\section{Other models and more realism in choice scenario}

Our analysis so far has made 3 assumptions. One is that the individual choices depend on the decisions made by others according to the choices of others using (1). This is an expression obtained from the first principles of estimation and also tested in sticklebacks (Pérez-Escudero and de Polavieja 2011). Our results, however, do not depend on this expression. Other expressions give similar results (Fig. 4, see Methods for mathematical expressions). We present results for a mathematical expression used for zebrafish and ants (Arganda et al. 2012) (rule in Fig. 4a, blue and result in Fig. $4 \mathrm{~b}$ ) and another expression used for theoretical analysis of collective decisions (rule in Fig. 4a, green and result in Fig. 4c). We also added a version of our rule in Eq. 1 but that saturates at $p_{\max }<1$ instead of 1 (Fig. $4 \mathrm{a}$, red) to show that in this case, the same result is obtained (Fig. $4 \mathrm{~d}$, $p_{\max }=0.9$.).

The other two assumptions, deciding by rounds and with strict order in each round, were used only for clarity of 
Fig. 3 Error propagation in large groups. a Probability that the first individual chooses the correct option in subsequent rounds given that the first two individuals chose incorrectly in the first round. Each color represents the probability for a given round (from second to fifth). b Evolution of the accuracy with rounds. We computed the proportion of individuals choosing the correct option at each round for group sizes of 4,8 , and 12
A
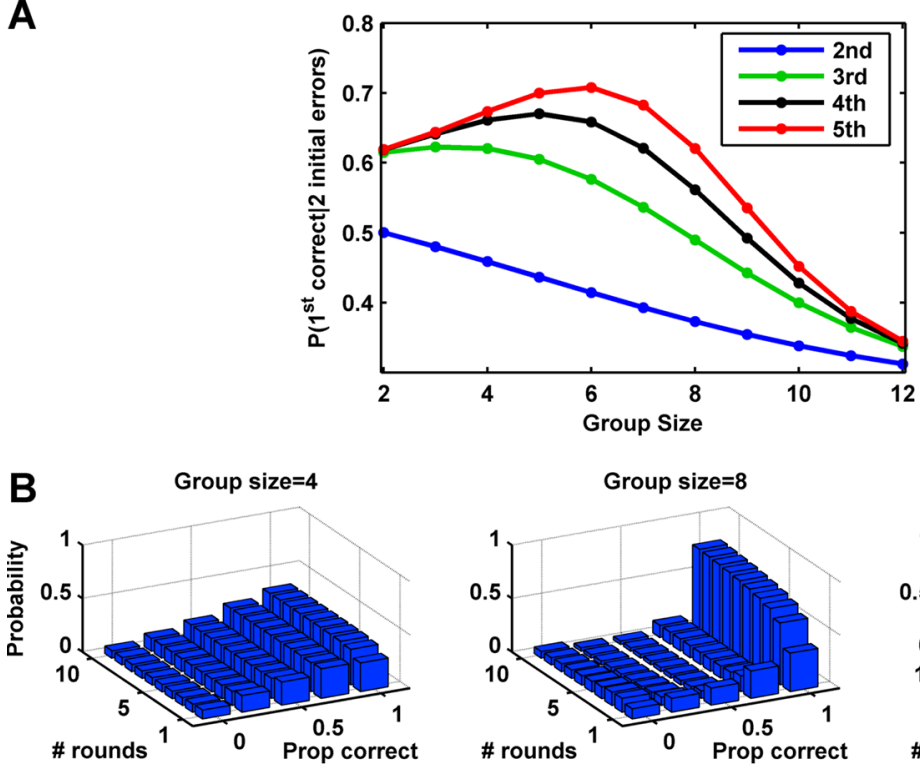

analysis and will not be adequate for most experiments. We found the same results for more stochastic versions of decision order eliminating one of the two assumptions or both assumptions (Fig. 5, see Methods for details).

Keeping rounds but choosing in random order in each round gives very similar results (Fig. 5a). We alsoconsidered a second scenario for the order of decisions in which agents

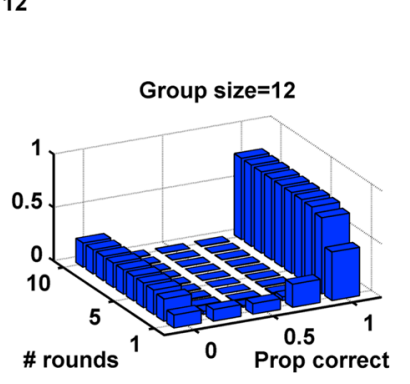

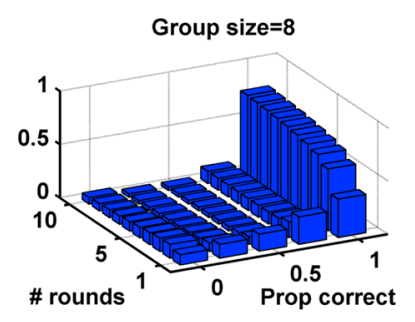

\section{(15)}

keep a similar order but with no strict rounds. This is a likely mechanism in polarized animal groups, where we know information flows from front to rear positions (see Herbert-Read et al. 2011; Katz et al. 2011). This model also gave very similar results (Fig. 5b, see Methods). Agents are ordered not by who chooses but by who has the highest probability of choosing. The actual order is then not strict
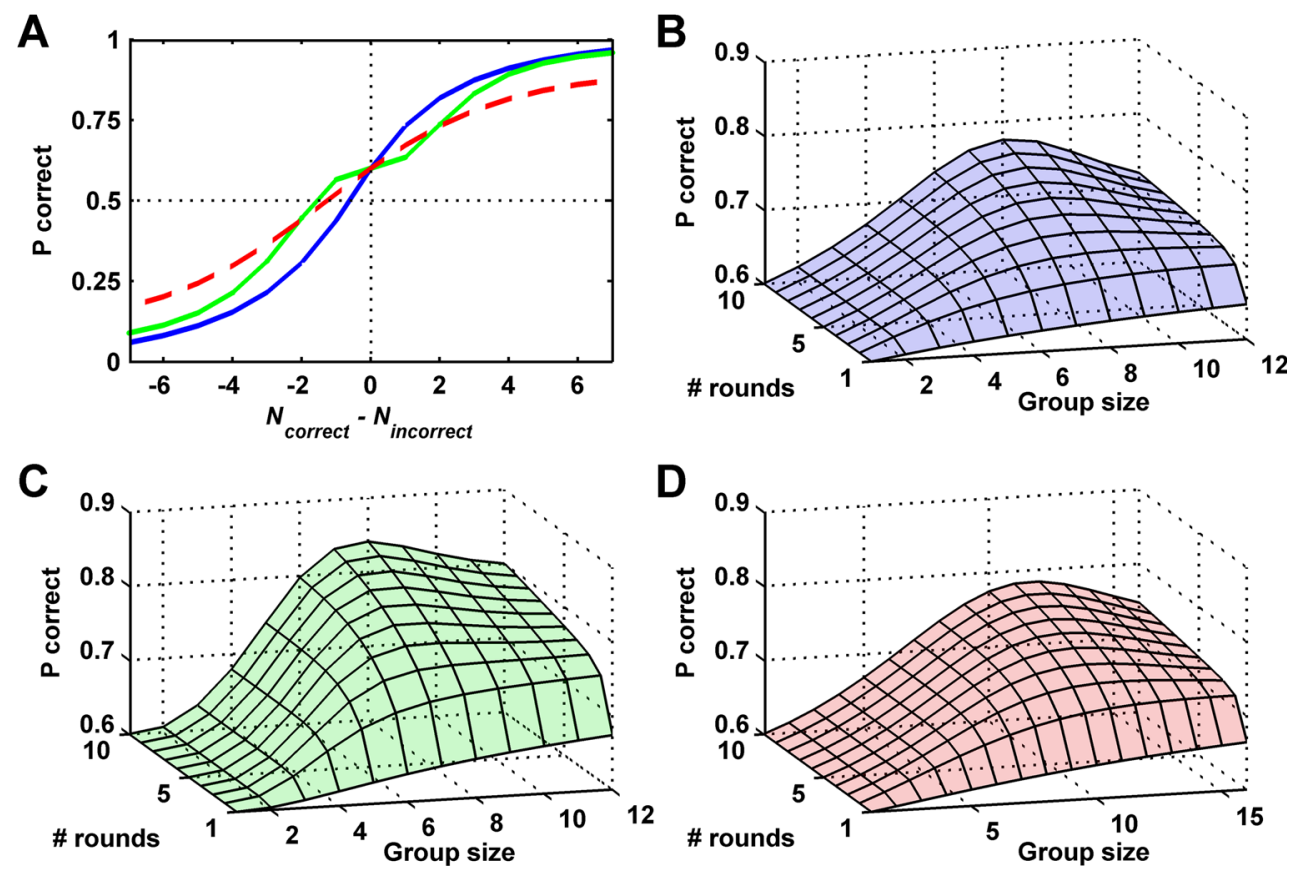

Fig. 4 Accuracy for different decision rules. a Examples of individual decisions rules with different probabilities of an individual choosing the correct option depending on how many more individuals have chosen that option. Blue: from Arganda et al. (2012), with parameters $a_{x}=2.5, a_{y}=1.33, k=0.5$, and $s=2$. Green: from Sumpter and Pratt (2009), with parameters $p_{x 00}=2 / 3, a=0.02, k=2.5$,

$T=10$, and $m=1$. Red: as Eq. 1 in the main text but converging to $p_{\max }=0.9$ and $p_{\min }=0.1$. See Methods for mathematical expressions. b Average probability of choosing correct option depending on group size and number of decision rounds when an individual follows the rule in blue in $\mathbf{a}$. $\mathbf{c}$ Same as $\mathbf{b}$ but for the rule in green in $\mathbf{a}$. $\mathbf{d}$ Same as $\mathbf{b}$ but using the rule in red in $\mathbf{a}$ 
Fig. 5 Different degrees of randomness in decision order. Results for decision formula in Eq. 1 in the main text with the same parameters as in Fig. 2 but with different ordering scenarios. See Methods for further details. a Randomized decision order where all individuals decide before re-evaluation. b Randomized order where each individual is ordered by a higher probability of decision. Probabilities are Gaussians centered at the order position of each individual and with standard deviation of two positions. c Completely random decisions. d Self-propelled particle simulations, where each individual has a probability of deciding at each iteration and Eq. 1 is applied using agents pointing to correct option $\left(N_{c}\right)$ and incorrect option $\left(N_{i}\right)$
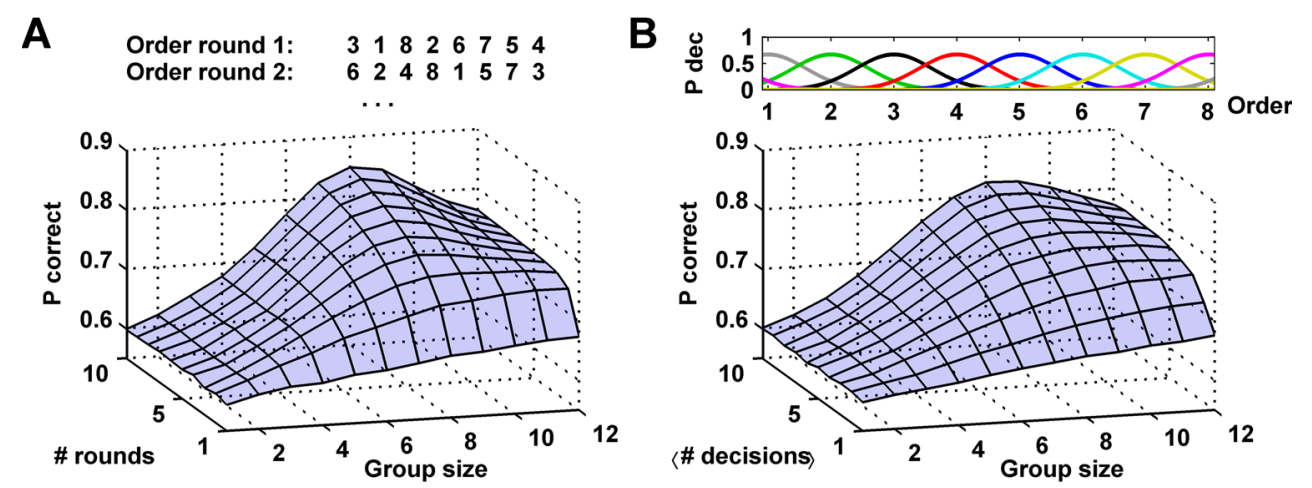

C
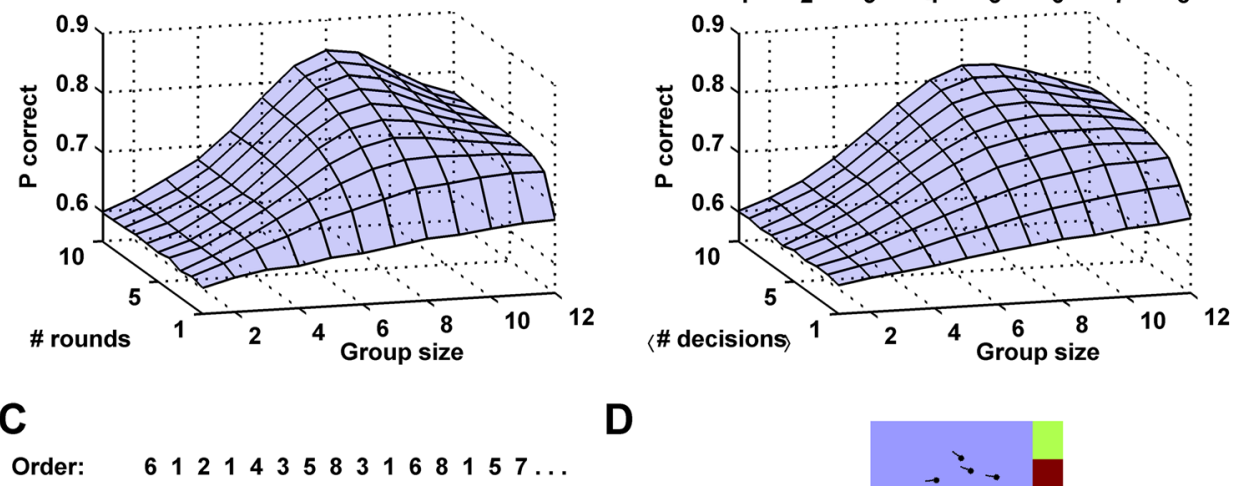

D

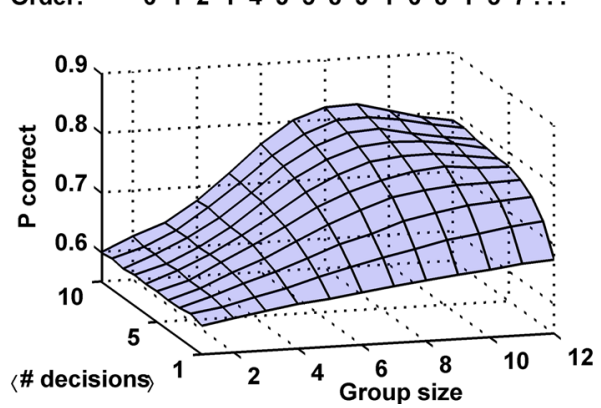

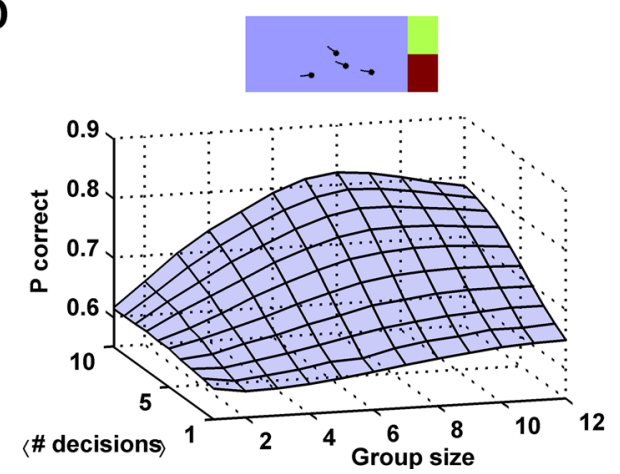

and there is then no clean separation in rounds in which each animal makes a decision. Instead, we order by number of average decisions per agent (Fig. 5b).

We also considered a completely random order with repetition possible that has then no predefined order and no rounds (Fig. 5c, see Methods). In this case, the highest accuracy is also at intermediate group sizes.

The last case we considered was one with agents moving in space and sensitive to the difference of animals moving towards one option and the other (see Methods). When agents are far from the place of choice, they point randomly to the two options, but when closer, they acquire some private information about which option is the best to choose. This case also predicts the highest accuracy at intermediate group sizes (Fig. 5d).

In all these cases, some individuals start to have access to social information before the others and in re-evaluation there is then a process by which all have access to it. Reevaluation then increases the amount of information animals have access to but also cascades of errors are more difficult to stop when reconsiderations take place in large groups.

\section{Local sampling version of the models}

So far, we have considered the case in which individuals are influenced by all the rest of the group. It is not necessary that individuals need to count precisely for this result, only that they follow influence curves like those of Fig. 4a. That is, for low numbers, they need to be more sensitive to choosing one option the more individuals are choosing that option and effectively saturate at larger numbers.

However, there are situations in which each individual is only influenced by a small number of individuals (Ballerini et al. 2008). We therefore studied the model as originally presented (Fig. 2a) but in which each individual is only influenced by a small number of individuals in the group (Fig. 6a, b).

For a given number of influencing individuals, $N_{l o c}$, the results up to this value are the same as before and after there is a slow increase of accuracy with group size. So if the value of the social parameter $s$ produced in previous calculations a peak after $N_{l o c}$, that peak is lost in the new calculation (Fig. 6a, $N_{l o c}=4$ and $s=2$ ). However, when the social parameter $s$ produced in previous calculations a peak before $N_{l o c}$, that peak remains in the new calculation (Fig. $6 \mathrm{~b}, N_{l o c}=6$ and $s=2$ ). In contrast to previous results, however, the accuracy of the group steadily increases with group size and for large enough values it wins over the local optimum at small group size. The result is not a monotonically increasing accuracy with group size, but also an additional local optimum size for small sizes. Figure $6 \mathrm{c}$, d gives analogous results but for $N_{l o c}=8$, with Fig. 6 d simply a wider set of values for group sizes.

We also studied the spatial model in Fig. $5 \mathrm{~d}$ but adding the restriction that each individual is only influenced by a subgroup (Fig. 6e, f). We used two different versions, one in which animals used the information coming from their 
Fig. 6 Accuracy when individuals are only influenced by a subgroup. a Results as for model in Fig. 5a but with the parameter $s=2$ and influence by $N_{l o c}=4$ individuals. b Same as a but for 6 individuals. $\mathbf{c}$ Same as a but for 8 individuals. d Same as $\mathbf{c}$ but studying the effect up to a group size of 32 individuals. e Simulations as in Fig. $5 \mathrm{~d}$ but when individuals use their 6 nearest neighbors for making the decisions. f Same as e but when individuals use all the individuals within a distance, plotted up to a group size of 32 individuals. See

"Methods" for details of models
A

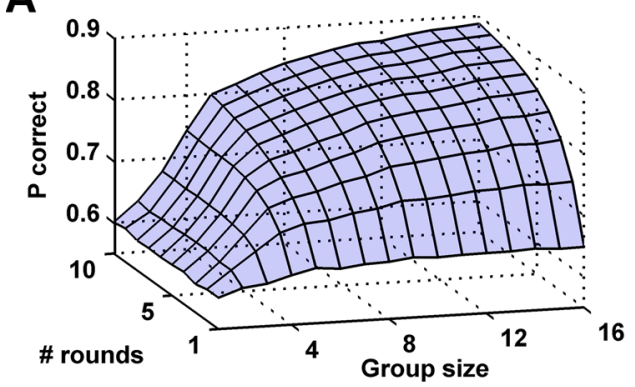

C

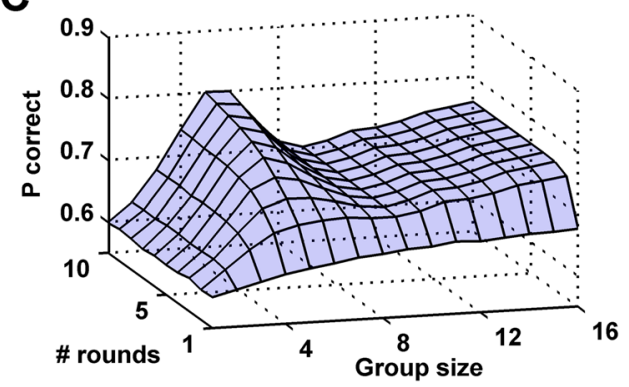

E

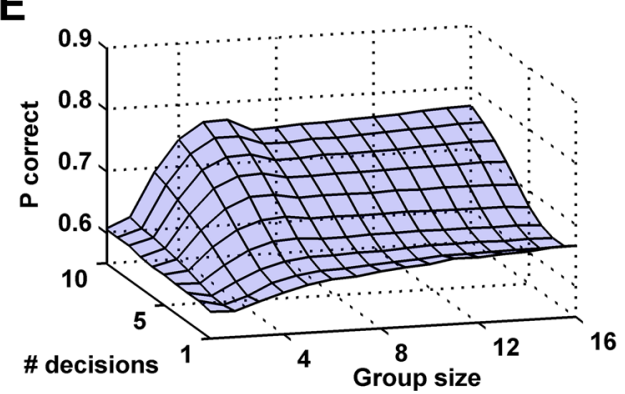

B

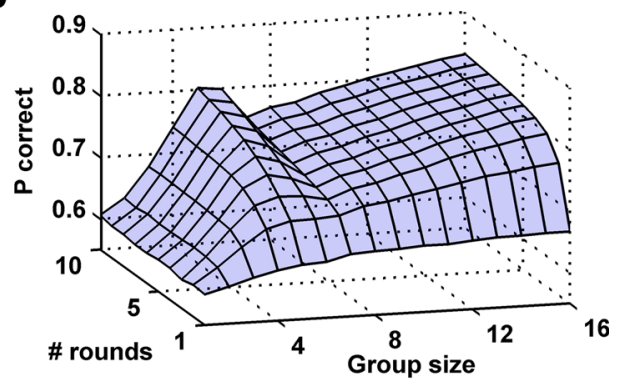

D

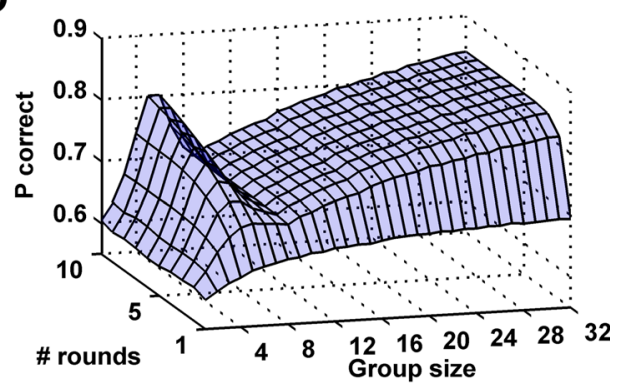

$\mathbf{F}$

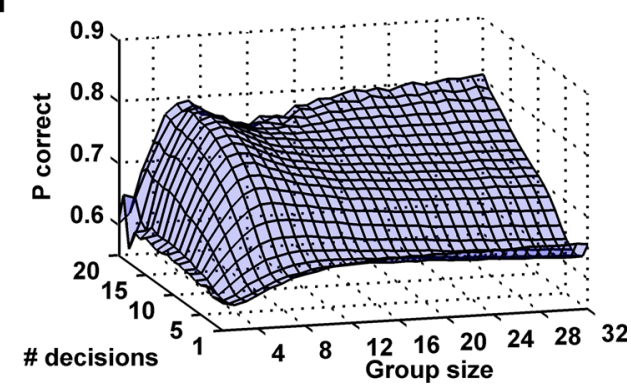

nearest neighbors (Fig. 6e) and another one in which they used the information of all the individuals within a given distance (Fig. 6f). The results of both scenarios again show a local optimum at small group size and a slow increase of accuracy for larger group sizes. See "Methods" for details of models and variants giving similar results.

\section{Discussion}

Decisions with re-evaluation point to the highest accuracy in groups of small or very large size. When an agent has access to all other agents, we found a maximal accuracy in small groups. In these cases, cascading of errors makes decisions for large groups more inaccurate on average. We have also seen that when each agent interacts with only a subset of other agents, error cascades are very unlikely to reach the whole group. This then results in high accuracy in small groups but also in very large groups.

Small group sizes are often observed (Avilés and Tufiño 1998; Brown and Brown 1996; Gerard et al. 2002; Williams et al. 2003; VanderWaal et al. 2009), but decision accuracy might be one among many factors determining the final group size (Brown 1982; Sibly 1967; Giraldeau and Gillis 1985; Higashi and Yamamura 1993; Krause and Ruxton 2002; Sumpter 2010), including foraging, migration, predation risk (Bertram 1980; Santema and Clutton-Brock 2013; da Silva and Terhune 1988), intra-specific competition (White 2008), and also the increase in the total amount of information available for the group (Galef and Wigmore 1983; Ward and Zahavi 1973).

When the other factors are more important in determining the group size, decision accuracy might then be suboptimal. We should in principle expect deviations from optimality to be more prevalent in the direction where the fitness decreases more slowly (Pèrez-Escudero et al. 2009). In this regard, our results show an asymmetry respect to the high accuracy group size at low group sizes. There is less of a loss in accuracy adding $N$ more animals to the group than subtracting them, favoring larger groups.

Another argument in favor for large group sizes is that the the size of small but accurate groups depends on the quality of private information and on the influence of the social information. If these factors change, agents would need to track them to be able to work at high accuracy. In contrast, accuracy at large group sizes depends less on these factors. 
When agents cannot track sufficiently well changes in private and social information, it is thus advantageous to work at large group sizes.

\section{Methods}

\section{Computing the probabilities of choosing the correct option}

We computed the probability of finding a given number of individuals choosing one option at the end of a given round from the exact probabilities of all the sequences that can produce this outcome, and then added them up. This was done for all cases where the order of the individual decisions was fixed (all figures except Fig. 5). For example, consider a group of 4 animals deciding in two rounds. Say that the sequence of decisions of the animals in the first round is $(y, x, y, y)$. The probability of choosing each option depends on the number of individuals that had chosen that option before. Therefore, the probability of this sequence at the first round is given by

$P^{1}(y, x, y, y)=P_{y}(0,0) P_{x}(0,1) P_{y}(1,1) P_{y}(1,2)$,

where $P_{y}\left(N_{x}, N_{y}\right)$ is the probability of choosing option $y$ given a number of individuals $N_{x}$ at option $x$ and $N_{y}$ at option $y$. Note that $P_{x}\left(N_{x}, N_{y}\right)=1-P_{y}\left(N_{x}, N_{y}\right)$. If the sequence of decisions at round 2 is $(y, x, y, y)$, where the third individual has changed its decision, the probability of the second round is

$$
P^{2}(y, x, y, y)=P_{y}(1,2) P_{x}(0,3) P_{y}(1,2) P_{y}(1,2) .
$$

The probability of the whole sequence is calculated as the product of $P^{1}(y, x, y, y)$ and $P^{2}(y, x, y, y)$. This would end up on a state in which 3 animals have chosen option $y$ and 2 have chosen option $x$ at the end of round 2, but there are more sequences generating the same final state. Therefore, to obtain the probability of a certain state, we must add the probabilities of all possible sequences that lead to this final state. Once we have the probabilities of each state, the probability of choosing the correct option per individual would be obtained by multiplying the probability of each state by the proportion of individuals that choose the correct option, and then adding these products through all the final states.

In cases where the individual decisions did not follow a fixed order, the previous algorithm to compute probabilities could not be applied. Instead, we simulated 50,000 (see "Different decision sequences" section for details).

\section{Different decision formulas}

We used alternatives to Eq. 1 when modeling the social influence of other individuals. In Fig. 4a (blue), we plot the expression from reference (Arganda et al. 2012),

$P_{y}=\left(1+\frac{1+a_{y} s^{-\left(N_{y}-k N_{x}\right)}}{1+a_{x} s^{-\left(N_{x}-k N_{y}\right)}}\right)^{-1}$.

This expression has two parameters for the private information, $a_{x}$ and $a_{y}$, and two parameter for the social influence, $s$ and $k$.

We also used the decision expression from reference Sumpter and Pratt (2009). For this model, the probability that option $y$ is good is given by

$p_{y}=p_{y 00}\left(a+(m-a) \frac{N_{y}^{k}}{T^{k}+N_{y}^{k}}\right)$,

with $p_{y 00}, a, m, T$, and $k$ parameters determining the shape of the function. A similar function determines the probability that $x$ is good, and the probability of choosing $y$ then is $P_{y}=p_{y} /\left(p_{y}+p_{x}\right)$, resulting in

$P_{y}=\left(1+\frac{p_{x 00}}{p_{y 00}} \cdot \frac{1+\left(\frac{m}{a}-1\right) \frac{N_{x}^{k}}{T^{k}+N_{x}^{k}}}{1+\left(\frac{m}{a}-1\right) \frac{N_{y}^{k}}{T^{k}+N_{y}^{k}}}\right)^{-1}$,

illustrated in Fig. 4a (green).

We also used an expression (1) of the main text but with a maximum probability of $P_{\max }$ and a minimum probability of $1-P_{\max }$, a s

$P_{y}=\left[\frac{1}{1+\frac{1}{a^{\prime}}\left(\frac{1}{s}\right)^{N_{y}-N_{x}}}\right]\left(2 P_{\max }-1\right)+\left(1-P_{\max }\right)$,

In the main text, we illustrated the case with $P_{\max }=$ 0.9 . The value of parameter $a^{\prime}$ that gives a 0.6 probability of choosing the correct option when there is no social information is $a^{\prime}=1.6666$.

\section{Different decision sequences}

For Fig. 5a, we randomized the order in which individuals decided at each round.

For Fig. 5b, we considered that each individual has a Gaussian probability of making a decision centered at its fixed turn and with $\sigma=1$. The Gaussians are added and the sum is normalized to compute the individual probabilities of making a decision at a given turn. The turns run in order from the first to the last individual and which individual decides at each turn is determined based on these probabilities. 
For Fig. 5c, the order of the decisions is completely random, so the decision could be made by any individual at each time step.

For Fig. 5d, we added a spatial component to the model. We created a two dimensional rectangular space $\left(x_{\max }=\right.$ $\left.50, y_{\max }=100\right)$ with two options of the same size $(25 \times 25)$ at the end of the $y$ dimension. We then simulated a dynamic decision-making process in which groups of individuals had to choose between the two options while moving towards them. The individuals started in a position given by a random uniform distribution centered at $(x=25, y=20)$ with a width of 5. Each individual randomly chooses to move towards one of two options for initialization. After that, each individual has a probability $p_{d e c}$ of making a decision at its corresponding turn, fixed before the simulations. In the simulations, we iterate through several values of $p_{d e c}(0.05-$ 0.4 ) in order to obtain different values for the number of decisions. If the individual makes a decision, it will choose one of the two options with a probability given by Eq. 1 . In order to avoid any spatial artifact, once an option is chosen, the individuals would randomly move towards one of the pixels of that option. If the individual does not make a decision, it will keep moving in the same direction as in the previous iteration. The number of individuals choosing each option would be counted and added to the decision equation, so the probabilities would be constantly updated. An individual would finish the trial when it reaches one of the options. If an individual reaches one of the options without having made any decision apart from the initialization one, it would not be taken into account for computing the accuracy. Once each simulation is finished, we compute both the number of decisions each individual has made and the final outcome of the simulation. We then use these numbers to compute the probabilities of choosing the correct option, averaged over 100,000 simulations.

\section{Decisions based on fewer individuals}

In Fig. 6a-d, each individual selects a random subgroup of $N_{\text {loc }}$ individuals, which are the ones used when computing the probabilities of going to each option for all decision rounds. These subgroups are not reciprocal and can overlap between individuals. As before, the results are averaged over 50,000 simulations.

We also tested other ways of selecting the subgroups of individuals, including (a) a randomization for each decision round, (b) selecting neighbors in the decision order instead of random individuals, and (c) using the different decision order protocols of Fig. 5. We always obtained results similar to those of Fig. $6 \mathrm{a}-\mathrm{d}$.

For the spatio-temporal simulations, we used two ways of selecting the subgroup of individuals. In Fig. 6e, for each decision, individuals use their nearest neighbors to compute the probabilities of going to each option. In Fig. 6f, the subgroup is defined by all individuals within a fixed radius $(r=7.5)$ from the deciding individual.

Acknowledgements We thank Carlos Ribeiro, Jacques Gautrais, members of the de Polavieja lab, and other members of the Champalimaud Neuroscience Programme for discussions. We acknowledge funding from Fundaçao para a Ciência e Tecnologia PTDC/NEUSCC/0948/2014 (to G.G.d.P.) and from Champalimaud Foundation (to G.G.d.P.).

Funding Information We acknowledge a JAE-CSIC fellowship (to J.V.P), a FPU fellowship from Ministerio de Economía y Competitividad, Spain (AP2006-01666 to A.P.-E.), funding from Spanish Plan Nacional BFU2009-09967, BFU2012-33448 and BFU201349512-EXP, Fundaçao para a Ciência e Tecnologia PTDC/NEU-SCC/ 0948/2014 (to G.G.d.P.) and Champalimaud Foundation (to G.G.d.P.).

Open Access This article is distributed under the terms of the Creative Commons Attribution 4.0 International License (http://creativecom mons.org/licenses/by/4.0/), which permits unrestricted use, distribution, and reproduction in any medium, provided you give appropriate credit to the original author(s) and the source, provide a link to the Creative Commons license, and indicate if changes were made.

\section{References}

Arganda S, Pérez-escudero A, Polavieja GGD (2012) A common rule for decision making in animal collective across species. Proc Nat Acad Sci 110(9):3651-3651

Avilés L, Tufiño P (1998) Colony size and individual fitness in the social spider Anelosimus eximius. Amer Nat 152(3):403-418

Ballerini M, Cabibbo N, Candelier R, Cavagna A, Cisbani E, Giardina I et al (2008) Interaction ruling animal collective behavior depends on topological rather than metric distance: evidence from a field study. Proc Nat Acad Sci USA 105(4):1232-7

Banerjee AV (1992) A simple model of herd behavior. Quart J Econ 107(2):797-817

Bertram BCR (1980) Vigilance and group size in ostriches. Animal Behaviour 28(1):278-286

Bikhchandani S, Hirshleifer D, Welch I (1992) A theory of fads, fashion, custom, and cultural change as informational cascades. J Polit Econ 100(5):992

Boccaletti S, Latora V, Moreno Y, Chavez M, Hwang DU (2006) Complex networks: structure and dynamics. Phys Rep 424(45):175-308

Bonabeau E, Dagorn L, Frèon P (1999) Scaling in animal group-size distributions. Proc Nat Acad Sci 96(8):4472-4477

Brown JL (1982) Optimal group size in territorial animals. J Theor Biol 95:793-810

Brown CR, Brown MB (1996) Coloniality in the cliff swallow: the effect of group size on social behavior. University of Chicago Press

Castellano C, Fortunato S, Loreto V, Castellano C, Fortunato S, Fortunato S (2009) Statistical physics of social dynamics. Rev Modern Phys 81(2):591-646

Cockburn A (1998) Evolution of helping behavior in cooperatively breeding birds. Ann Rev Ecol Syst 29:141-177

Condorcet JANdC (1785) Essai sur l'application de l'analyse à la probabilité des décisions rendues à la pluralité des voix

da Silva J, Terhune JM (1988) Harbour seal grouping as an antipredator strategy. Animal Behaviour 36(5):1309-1316 
Galef BG, Wigmore SW (1983) Transfer of information concerning distant foods: a laboratory investigation of the 'informationcentre' hypothesis. Animal Behaviour 31:748-758

Galton F (1907) Vox populi. Nature 75:450-451

Gerard JF, Bideau E, Maublanc ML, Loisel P, Marchal C (2002) Herd size in large herbivores. Encoded in the individual or emergent? Biol Bull 202:275-282

Giraldeau LA, Gillis D (1985) Optimal group size can be stable: a reply to Sibly. Animal Behaviour 33:666-667

Havlin S, Bunde A (1991) Fractals and disordered systems. Springer, New York

Herbert-Read JE, Perna A, Mann RP, Schaerf TM, Sumpter DJT, Ward AJW (2011) Inferring the rules of interaction of shoaling fish. Proc Nat Acad Sci USA 108(46):18726-31

Higashi M, Yamamura N (1993) What determines animal group size? Insider-outsider conflict and its resolution. Amer Natur 142(3):553-563

Kao AB, Couzin ID, PRS B (2014) Decision accuracy in complex environments is often maximized by small group sizes. Decision accuracy in complex environments is often maximized by small group sizes. Proc R Soc B

Katz Y, Tunstrom K, Ioannou CC, Huepe C, Couzin ID (2011) Inferring the structure and dynamics of interactions in schooling fish. Proc Nat Acad Sci 1-9

Krause J, Ruxton GD (2002) Living in groups. Oxford series in ecology and evolution. Oxford University Press

Madirolas G, de Polavieja GG (2015) Improving collective estimations using resistance to social influence. PLoS Comput Biol 11(11)

Niwa H (1998) School size statistics of fish. J Theor Biol 195(3):351-61

Packer C, Scheel D, Pusey AE (1990) Why lions form groups: food is not enough. Amer Natur 130(1):526-543

Pérez-Escudero A, de Polavieja GG (2011) Collective animal behavior from Bayesian estimation and probability matching. PLoS Comput Biol 7(11):e1002282
Pèrez-Escudero A, Rivera-Alba M, de Polavieja GG, PèRez-Escudero A, Rivera-Alba M, de Polavieja GG (2009) Structure of deviations from optimality in biological systems. Proc Nat Acad Sci 106(48):20544-20549

Santema P, Clutton-Brock T (2013) Meerkat helpers increase sentinel behaviour and bipedal vigilance in the presence of pups. Animal Behaviour 85(3):655-661

Shao J, Havlin S, Stanley HE (2009) Dynamic opinion model and invasion percolation. Phys Rev Lett. 103(1)

Sibly RM (1967) Optimal group size is unstable. Animal behaviour 31(3):1-2

Stauffer D, Aharony A (1994) Introduction to percolation theory

Sumpter DJT (2010) Collective animal behavior. Princeton University Press, Princeton. Monographs in Behavior \& Ecology

Sumpter DJT, Pratt SC (2009) Quorum responses and consensus decision making. Philosl Trans R Soc London Series B Biol Sci 364:743-753

Sumpter DJT, Krause J, James R, Couzin ID, Ward AJW (2008) Consensus decision making by fish. Curr Biol 18(22): $1773-7$

Surowiecki J (2004) The wisdom of crowds. Random House Inc.

VanderWaal KL, Mosser A, Packer C (2009) Optimal group size, dispersal decisions and postdispersal relationships in female African lions. Animal Behaviour 77(4):949-954

Ward P, Zahavi A (1973) The importance of certain assemblages of birds as "information-centres" for food-finding. Ibis 115:517534

Ward AJW, Sumpter DJT, Couzin ID, Hart PJB, Krause J (2008) Quorum decision-making facilitates information transfer in fish shoals. Proc Nat Acad Sci USA 105(19):6948-53

White TCR (2008) The role of food, weather and climate in limiting the abundance of animals. Biol Rev 83:227-248

Williams CK, Lutz RS, Applegate RD (2003) Optimal group size and northern bobwhite coveys. Anim Behaviour 66:377-387 